\title{
On collisional energy transfer in recombination and dissociation reactions: A Wiener-Hopf problem and the effect of a near elastic peak
}

\author{
Zhaoyan Zhu and R. A. Marcus ${ }^{\text {a) }}$ \\ Noyes Laboratory of Chemical Physics, California Institute of Technology, \\ Pasadena, California 91125, USA
}

(Received 12 June 2008; accepted 22 October 2008; published online 2 December 2008)

\begin{abstract}
The effect of the large impact parameter near-elastic peak of collisional energy transfer for unimolecular dissociation/bimolecular recombination reactions is studied. To this end, the conventional single exponential model, a biexponential model that fits the literature classical trajectory data better, a model with a singularity at zero energy transfer, and the most realistic model, a model with a near-singularity, are fitted to the trajectory data in the literature. The typical effect of the energy transfer on the recombination rate constant is maximal at low pressures and this region is the one studied here. The distribution function for the limiting dissociation rate constant $k_{0}$ at low pressures is shown to obey a Wiener-Hopf integral equation and is solved analytically for the first two models and perturbatively for the other two. For the single exponential model, this method yields the trial solution of Troe. The results are applied to the dissociation of $\mathrm{O}_{3}$ in the presence of argon, for which classical mechanical trajectory data are available. The $k_{0}$ 's for various models are calculated and compared, the value for the near-singularity model being about ten times larger than that for the first two models. This trend reflects the contribution to the cross section from collisions with larger impact parameter. In the present study of the near-singularity model, it is found that $k_{0}$ is not sensitive to reasonable values for the lower bound. Energy transfer values $\langle\Delta E\rangle$ 's are also calculated and compared and can be similarly understood. However, unlike the $k_{0}$ values, they are sensitive to the lower bound, and so any comparison of a classical trajectory analysis for $\langle\Delta E\rangle$ 's with the kinetic experimental data needs particular care. (C) 2008 American Institute of Physics.
\end{abstract}

[DOI: $10.1063 / 1.3026605]$

\section{INTRODUCTION}

In the treatment of gas phase dissociation, unimolecular isomerization, and bimolecular recombination reactions, it has been recognized for many years that "weak collisions" rather than "strong collisions" play a major role in the activation and deactivation of the vibrationally hot intermediate complexes in these reactions. ${ }^{1-5}$ Our interest in the subject was prompted by studies of ozone whose formation and isotopic effects have been of much recent interest. ${ }^{6-22}$ In general, the formation of a molecule $A B$ is described by

$$
\begin{aligned}
& A+B \rightleftarrows A B^{*}, \\
& A B^{*}+M \rightarrow A B+M,
\end{aligned}
$$

where $M$ is a collision partner and $A B^{*}$ is a vibrationally excited intermediate. In a weak collision assumption, unlike in a strong collision one, many collisions with $M$ are required to activate and deactivate a reactant molecule. When the collision is "weak," the $A B^{*}$ may still have enough energy after the collision in reaction (2) to redissociate into $A+B$, instead of always being "deactivated," and so a set of such equations with different energy is considered, leading to a master equation or to a steady-state equation. The latter is then solved for the probability distribution function for the vibrational energy in the energetic intermediate $A B^{*}$.

${ }^{a)}$ Electronic mail: ram@caltech.edu.
Information on the collisional energy transfer in reactions such as in Eq. (2) is usually obtained from the pressure dependence of the reaction rate of the overall reactions (1) and (2), using the solution of the collisional master or steadystate equation to fit these experimental reaction rate versus pressure data. ${ }^{3,5}$ To this end, a functional form for the collision energy transfer probability, denoted here by $Z\left(E^{\prime}, E\right)$, is typically assumed, and its parameters are calculated from the fit. The functional forms used for this purpose are usually the exponential model introduced by Rabinovitch, used in Sec. II A, or a step ladder in which the reactant molecule gains or loses energy in collisions in discrete amounts called "steps."1,2,23,24 Hold et al. ${ }^{25}$ also introduced a stretched exponential model. A biexponential model was used by Brown and Miller ${ }^{26}$ and modified by Hu and Hase. ${ }^{27}$ Complementing these studies have been $a b$ initio or semiempirical calculations of the collisional energy transfer, frequently using classical mechanical trajectories for the collisions. ${ }^{26-36}$ Analytical treatments of vibrational energy transfer have been given for particular cases. ${ }^{5,34-38}$ In particular, a detailed discussion of the original master equation and of its steady-state approximation is given by Penner and Forst, ${ }^{38}$ who expressed the solution in terms of hypergeometric functions.

The $Z\left(E^{\prime}, E\right)$ is defined as the number of collisions per unit time with energy transfer for the vibrationally excited intermediate $E \rightarrow\left(E^{\prime}, E^{\prime}+d E^{\prime}\right)$ per unit $d E^{\prime} . Z\left(E^{\prime}, E\right)$ has units of $\mathrm{s}^{-1}$ energy ${ }^{-1}$ when it is chosen to be the product of 

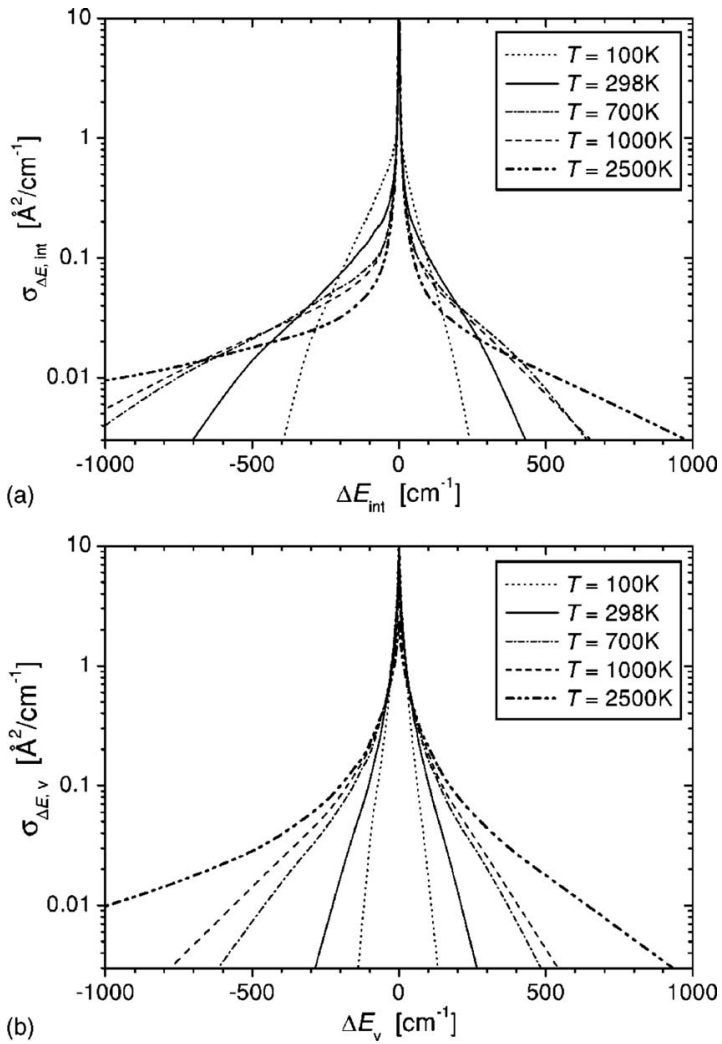

FIG. 1. Cross sections for internal (a) and vibrational (b) energy transfers as functions of $\Delta E_{\text {int }}$ and $\Delta E_{v}$ for different temperatures (Ref. 40).

the concentration of colliders and the bimolecular collision rate constant for the transition $E \rightarrow E^{\prime}$ per $d E^{\prime}$. Its theoretical calculation involves an integration over impact parameters $b$, using $2 \pi b d b$ as a weighting factor. Collisions with large $b$ contribute mainly to the energy transfer near $E^{\prime}-E \approx 0$. When plotted versus $E^{\prime}-E$, they yield an elastic-collision peak in the classical limit at $E=E^{\prime}$ corresponding to $b=\infty$. The larger the average value of the energy transfer per collision in any reaction, the further the important energy transfer region is from the elastic $E^{\prime}-E=0$ peak. Examples of the tendency toward a singular behavior at $E^{\prime}=E$ are seen in Refs. 39-43. The data of Ivanov and Schinke ${ }^{40}$ shown in Fig. 1 are used later as an example. From a quantum mechanical view, the inelastic collisions have a lower bound for the energy transfer $\left|E-E^{\prime}\right|$, namely, a quantum of rotational or vibrational energy, depending upon the collision. So in quantum mechanical calculations, there is no such singularity, but instead there is a near-elastic peak. Therefore, a lower bound should be imposed. A maximum impact parameter $b_{\max }$ such that no trajectories lead to sizable energy transfer for $b>b_{\text {max }}$ has usually been imposed in the literature to avoid this peak. ${ }^{26-35}$ Then a single exponential, step-ladder or biexponential model was usually adopted to fit the trajectory data. For example, Brown and Miller ${ }^{26}$ neglected the bin in which $E^{\prime}-E \approx 0$ with a bin size of around $30 \mathrm{~cm}^{-1}$ in a biexponential fit to the trajectory data. $\mathrm{Hu}$ and $\mathrm{Hase}^{27}$ suggested that $b_{\max }$ should be identified as the value of $b$ at which the average energy transfer equals the inverse of the state density. In such a choice, the resulting collision cross section was considerably larger than the usually assumed value, but within $5 \%$ of the experimental value. ${ }^{27}$

The paper is organized as follows. The theory is described in Sec. II for the different models. It is applied to a particular system in Sec. III. The results are discussed in Sec. IV, with concluding remarks in Sec. V.

\section{THEORY}

\section{A. General aspects}

In using trajectories to calculate the transition rate $Z\left(E^{\prime}, E\right)$, a random sampling of trajectories is performed over the vibrational and rotational coordinates and their conjugate momenta of the vibrationally excited intermediate and over a Boltzmann-weighted distribution of relative velocities of the collision partners. ${ }^{31-36}$ The calculations of energy transfer are typically made as a function of the internal energy $E$ of the energetic intermediate, its total angular momentum $J,{ }^{44}$ and occasionally $K$, the projection of $J$ along a specified principal axis of rotation, typically that with the smallest moment of inertia. For notational simplicity, we suppress the symbol $J$ in the following.

To obtain insight into the effect of the near-elastic peak at $\left|E^{\prime}-E\right|=0$ in the comparison between experimental data and trajectories, it is convenient to consider the collisional steady-state/reaction equations, and obtain approximate analytical solutions. Examples of other treatments are also available. $^{45-59}$

We focus on the limiting low pressure rate constant $k_{0}$. It is of particular interest partly because it describes the maximum effect of the collisions and partly because it is simpler to treat than the rate constant at higher pressures, where a solution of the complete master equation would have been necessary. While simple theoretical expressions for the distribution function of different $(E, J)$ states of the reactants and for the energy transfer can also be obtained for the highpressure limit of $k, k_{\infty}$, they do not provide insight into the effect of energy transfer on $k$ itself, since $k_{\infty}$ is independent of $Z\left(E^{\prime}, E\right)$. The effect of the near-elastic peak should be largest at low pressures, since the average energy of the reacting vibrationally excited intermediate in a unimolecular reaction or a bimolecular recombination is well known to decrease when the pressure is decreased. ${ }^{60}$ Accordingly, the vibrational energy of the typical molecule is closer to the energy dividing line between stable and unstable intermediates and so is closer to the near-elastic peak when the pressure is decreased.

To treat the kinetics for the recombination of two species $A+B \rightarrow A B$, one can either proceed from the reactants $(A+B)$ or from the product $(A B)$. If a tagged $A$ is followed in time in its progress to form $A B$, and if a tagged $A$ in $A B$ is followed in time in its progress to form $A+B$, beginning with an equilibrium concentrations of $A B$ for the given $A$ and $B$ concentration, the sum of the tagged distribution functions at any energy $E$ is equal to the equilibrium distribution at that $E$. Thus, to solve the problem, one can either begin with a tagged $A$ or a tagged $A B$. To simplify the comparison with earlier work, ${ }^{46-50}$ we begin with $A B$ and use the result to calculate also the rate of recombination $A+B \rightarrow A B$. 
We consider the reaction in the low pressure regime

$$
\begin{gathered}
M+A B(E) \underset{Z\left(E, E^{\prime}\right)}{\leftarrow} M+A B^{*}\left(E^{\prime}\right), \\
A B^{*}\left(E^{\prime}\right) \rightarrow A+B \quad\left(\text { if } E^{\prime} \geq 0\right),
\end{gathered}
$$

where $E^{\prime} \geq 0$ denotes the internal energies of any reaction intermediate $A B^{*}$ that can dissociate into the separated reactants without a further collision. Energies $E^{\prime}$ in the reactant that are negative are insufficient for dissociation.

The scheme [Eqs. (3) and (4)] is appropriate only for the low pressure limit of the dissociation rate. At higher pressures, an $A B^{*}\left(E^{\prime}\right)$ on a second collision can also yield an $A B^{*}$ with a different $E^{\prime}$, where both $E$ 's are greater than zero. At sufficiently low pressures, each $A B^{*}\left(E^{\prime}\right)$ formed in reaction (3) with $E^{\prime} \geq 0$ ultimately dissociates into $A+B$ before any further collision. For this case, the problem simplifies and a large set of equations (the master equation) for $A B^{*}\left(E^{\prime}\right)$ is not needed for $E^{\prime}>0$.

We denote the probability energy distribution function for $A B$ by $g(E)$ and write

$$
k_{0}=\int_{E^{\prime}=0}^{\infty} \int_{E=-\infty}^{0} g(E) Z\left(E^{\prime}, E\right) d E^{\prime} d E
$$

The equilibrium probability that $A B$ has an energy in the range $E, E+d E$ is $g_{\text {eq }}(E) d E$, where

$$
g_{\text {eq }}(E)=\rho(E) \exp (-E / k T) / Q
$$

and $\rho(E)$ denotes the density of quantum states of the molecule, $Q$ is the partition function of $A B$ in the center-of-mass system of coordinates. When the energy is measured relative to $E=0$, the dissociation limit $Q$ is also calculated relative to that energy, and so contains a factor $\exp (D / k T)$, where $D$ is the dissociation energy of $A B$ measured from the bottom of its potential well to the dissociation level. When $E$ becomes very negative in the steady-state problem, i.e., when $E \rightarrow-D$ and $g(E) \rightarrow g_{\text {eq }}(E)$. This condition on $g(E)$ serves as a boundary condition,

$$
g(E) \rightarrow g_{\text {eq }}(E) \quad E \rightarrow-D .
$$

After a short initial period, $g(E)$ relaxes toward a steady state. ${ }^{5}$ We use a steady-state approximation here. The steadystate equation for $g(E)$ is

$0=\int_{-\infty}^{0} Z\left(E, E^{\prime}\right) g\left(E^{\prime}\right) d E^{\prime}-g(E) \int_{-\infty}^{\infty} Z\left(E^{\prime}, E\right) d E^{\prime} \quad E \leq 0$.

The latter can be rewritten as

$$
g(E)=\int_{-\infty}^{0} Z\left(E, E^{\prime}\right) g\left(E^{\prime}\right) d E^{\prime} / \int_{-\infty}^{\infty} Z\left(E^{\prime}, E\right) d E^{\prime}, \quad E \leq 0 .
$$

An analytical solution of this integral equation for a single exponential model for the energy transfer was first given by Troe, ${ }^{5}$ who obtained it using a trial solution method. For the present article, we note instead that Eq. (7b) is a homogeneous Wiener-Hopf equation of the second kind and use the Wiener-Hopf method ${ }^{61}$ to obtain a solution.

We first study the single exponential and biexponential models with this method. For the single exponential model, in terms of a deactivation constant $\gamma$ and of an activation constant $\gamma^{\prime}$, we have

$$
\begin{aligned}
& Z\left(E^{\prime}, E\right)=Z_{0} \exp \left(-\left(E-E^{\prime}\right) / \gamma\right), \quad E^{\prime} \leq E, \\
& Z\left(E^{\prime}, E\right)=Z_{0} \exp \left(-\left(E^{\prime}-E\right) / \gamma^{\prime}\right), \quad E^{\prime} \geq E,
\end{aligned}
$$

and for the biexponential model,

$$
\begin{aligned}
Z\left(E^{\prime}, E\right)= & Z_{0}\left[\exp \left(-\left(E-E^{\prime}\right) / \gamma\right)\right. \\
& \left.+c \exp \left(-\left(E-E^{\prime}\right) / d\right)\right], \quad E^{\prime} \leq E, \\
Z\left(E^{\prime}, E\right)= & Z_{0}\left[\exp \left(-\left(E^{\prime}-E\right) / \gamma^{\prime}\right)\right. \\
& \left.+c \exp \left(-\left(E^{\prime}-E\right) / d^{\prime}\right)\right], \quad E^{\prime} \geq E,
\end{aligned}
$$

where $Z_{0}$ is a constant and $\gamma, \gamma^{\prime}$ and $d, d^{\prime}$ are related by microscopic reversibility (detailed balance).

The quantities $Z\left(E^{\prime}, E\right)$ and $Z\left(E, E^{\prime}\right)$ satisfy microscopic reversibility

$$
\rho(E) Z\left(E^{\prime}, E\right)=\rho\left(E^{\prime}\right) Z\left(E, E^{\prime}\right) \exp \left(-\left(E^{\prime}-E\right) / k T\right) .
$$

For practical purposes, we can typically treat the lower limit on $E$ as $E \rightarrow-\infty$, a minor approximation when $D \gg k T$. Neglecting the effect of the change in $\rho(E)$ between $E$ and $E^{\prime}$ in the vicinity of $E=0$, Eqs. (8a), (8b), (9a), (9b), and (10), yield

$$
\begin{aligned}
& \frac{1}{\gamma^{\prime}}=\frac{1}{\gamma}+\frac{1}{k T}, \\
& \frac{1}{d^{\prime}}=\frac{1}{d}+\frac{1}{k T} .
\end{aligned}
$$

The constants $c$ and $d$ are obtained later from fitting classical trajectory calculations data for vibrational energy transfer.

We have also examined a model with singularity at $E^{\prime}-E=0$ using another method,

$Z\left(E^{\prime}, E\right)=Z\left[1+C\left(E-E^{\prime}\right)^{-\alpha}\right] \exp \left(-\left(E-E^{\prime}\right) / \gamma\right), \quad E^{\prime} \leq E$,

$Z\left(E^{\prime}, E\right)=Z\left[1+C\left(E^{\prime}-E\right)^{-\alpha}\right] \exp \left(-\left(E^{\prime}-E\right) / \gamma^{\prime}\right), \quad E^{\prime} \geq E$,

where $\gamma$ and $\gamma^{\prime}$ are the same as those of the single exponential model. $C$ and $\alpha$ and are obtained later from classical trajectory data.

\section{B. Single exponential model}

To compare with the earlier and insightful result in literature by Troe, ${ }^{5}$ we use the single exponential expression for the collisional energy transfer rate, namely, Eqs. (8a) and (8b). The average "up-energy" transfer, defined as the average energy increase in the molecule for collisions that lead to an increase in energy, is 


$$
\begin{aligned}
\langle\Delta E\rangle_{\mathrm{up}} & =\int_{E}^{\infty}\left(E^{\prime}-E\right) Z\left(E^{\prime}, E\right) d E^{\prime} / \int_{E}^{\infty} Z\left(E^{\prime}, E\right) d E^{\prime} \\
& =\gamma^{\prime}, \quad E^{\prime} \geq E .
\end{aligned}
$$

Similarly the average "down"-energy transfer is

$$
\begin{aligned}
\langle\Delta E\rangle_{\text {down }} & =\int_{-\infty}^{E}\left(E^{\prime}-E\right) Z\left(E^{\prime}, E\right) d E^{\prime} / \int_{-\infty}^{E} Z\left(E^{\prime}, E\right) d E^{\prime} \\
& =-\gamma, \quad E^{\prime} \leq E .
\end{aligned}
$$

These quantities $\langle\Delta E\rangle_{\text {up }}$ and $\langle\Delta E\rangle_{\text {down }}$ are not observables in the usual reaction rate experiments and so cannot be directly compared with experiment. In computation, the quantity $\left\langle\Delta E^{2}\right\rangle$ is a more convenient parameter than $\langle\Delta E\rangle .{ }^{62,63}$ Here in our discussion, the average over impact parameter $b$ and the other initial variables is included in the definition of $Z\left(E^{\prime}, E\right)$ at the given $E$. The rate constant $k$ on the other hand, as a function of pressure and its limiting value at low pressure $k_{0}$, are the observables in these experiments. Nevertheless, since the values of the moments are often calculated in the literature from classical trajectories or from approximate fits to those data, the values of these moments are often cited, and are calculated here, bearing in mind that they are not directly observable and are model dependent.

To solve Eq. (7b), we use a Wiener-Hopf procedure ${ }^{61}$ and, as in the standard procedure, first extend the domain in Eqs. (7a) and (7b) from $E \leq 0$ to $E>0$ by introducing the functions $g_{-}(E)$ and $g_{+}(E), g(E)=g_{-}(E)+g_{+}(E)$, with the properties

$$
\begin{aligned}
& g_{-}(E)=g(E), \quad E \leq 0, \\
& =0, \quad E>0 \text {, } \\
& g_{+}(E)=0, \quad E \leq 0, \\
& =g(E), \quad E>0 \text {. }
\end{aligned}
$$

Here, $g(E)$ is the unknown function. From Eqs. (7b) and (14), we then obtain for the entire $E$-range, $-\infty<E<\infty$,

$$
\begin{aligned}
g_{-}(E)+ & g_{+}(E) \\
= & \int_{-\infty}^{\infty} g_{-}\left(E^{\prime}\right) Z\left(E, E^{\prime}\right) d E^{\prime} / \int_{-\infty}^{\infty} Z\left(E^{\prime}, E\right) d E^{\prime}, \\
& -\infty<E<\infty .
\end{aligned}
$$

For $E \leq 0$, this equation becomes

$$
\begin{aligned}
g_{-}(E)= & \int_{-\infty}^{0} g_{-}\left(E^{\prime}\right) Z\left(E, E^{\prime}\right) d E^{\prime} / \int_{-\infty}^{\infty} Z\left(E^{\prime}, E\right) d E^{\prime}, \\
& -\infty<E \leq 0,
\end{aligned}
$$

which coincides with Eq. (7b) for $E \leq 0$. For $E>0$, we have

$$
\begin{aligned}
& g_{+}(E)=\int_{-\infty}^{0} g_{-}\left(E^{\prime}\right) Z\left(E, E^{\prime}\right) d E^{\prime} / \int_{-\infty}^{\infty} Z\left(E^{\prime}, E\right) d E^{\prime}, \\
& 0<E<\infty .
\end{aligned}
$$

The idea behind the Wiener-Hopf method is to solve this pair of equations for $g_{+}(E)$ and $g_{-}(E)$ and hence, from Eq. (14), for $g(E)$. Because $Z\left(E, E^{\prime}\right)$ has one form in Eq. (8) when $E^{\prime} \geq E$ and has a different form when $E^{\prime} \leq E$, there are two terms for $Z\left(E, E^{\prime}\right)$. On taking the Fourier transform $\widetilde{f}(z)=\int_{-\infty}^{\infty} \mathrm{e}^{2 \pi i z E} f(E) d E, z=u+i v$, where $u$ and $v$ are real, and using the convolution theorem, we have from Eqs. (8) and (15a),

$$
\widetilde{g}_{-}(z)+\widetilde{g}_{+}(z)=\frac{\widetilde{g}_{-}(z)}{\gamma+\gamma^{\prime}}\left(\frac{1}{1 / \gamma+2 \pi i z}+\frac{1}{1 / \gamma^{\prime}-2 \pi i z}\right),
$$

which can be rewritten as

$$
\frac{2 \pi i z(2 \pi i z-1 / k T) \tilde{g}_{-}(z)}{1 / \gamma+2 \pi i z}=\frac{\left(1 / \gamma^{\prime}-2 \pi i z\right) \tilde{g}_{+}(z)}{\left(\gamma+\gamma^{\prime}\right)} .
$$

The solution for the $\widetilde{g}_{-}(z)$ in Eq. (17) obtained in Appendix A is

$$
\tilde{g}_{-}(z)=G_{s} \frac{(1 / \gamma+2 \pi i z)}{2 \pi i z(2 \pi i z-1 / k T)},
$$

where $G_{s}$ is a constant. The solution for $\widetilde{g}_{+}(z)$ is not needed but is given for completeness in Appendix A. The inverse transformation of Eq. (18) for $\widetilde{g}_{-}(z)$ yields

$$
g_{-}(E)=\int_{-\infty+i v}^{\infty+i v} \widetilde{g}_{-}(z) \mathrm{e}^{-2 \pi i z E} d z=G_{s}^{\prime}\left(\frac{k T}{\gamma^{\prime}} \mathrm{e}^{-E / k t}-\frac{k T}{\gamma}\right),
$$

where $G_{s}^{\prime}$ is a constant to be determined. We have from Eq. (14a) that $g_{-}(E)=g(E)$ for $E \leq 0$ and when $E \rightarrow-\infty$, we have $g(E) \rightarrow g_{\text {eq }}(E)$. Writing $g_{\text {eq }}(E)$ as $g_{\text {eq }}(0) \mathrm{e}^{(-E / k T)}$, a value is obtained for the constant $G_{s}^{\prime}, G_{s}^{\prime}=g_{\text {eq }}(0) \gamma^{\prime} / k T$, and hence

$$
g(E)=g_{\mathrm{eq}}(0)\left(\mathrm{e}^{-E / k T}-\frac{\gamma^{\prime}}{\gamma}\right),
$$

which is the trial solution given by Troe. ${ }^{5}$ Using it, the analytical solution for the low pressure "three-body" recombination rate constant $k_{0}$ can be obtained. From Eqs. (5) and (20),

$$
k_{0}=Z_{0} g_{\text {eq }}(0) \gamma \gamma^{\prime}\left(1-\frac{\gamma^{\prime 2}}{\gamma^{2}}\right)=Z_{0}\left(\gamma+\gamma^{\prime}\right) \frac{\gamma^{\prime 2} \rho(0)}{Q k T},
$$

where $Q$ contains the factor $\exp (D / k t)$. This result agrees with the trial solution of Troe. ${ }^{5}$

\section{Biexponential model}

In this model, the average up-energy transfer is given by

$$
\langle\Delta E\rangle_{\mathrm{up}}=\int_{E^{\prime}=E}^{\infty}\left(E^{\prime}-E\right) Z\left(E^{\prime}, E\right) d E^{\prime} / \int_{E^{\prime}=E}^{\infty} Z\left(E^{\prime}, E\right) d E^{\prime}=\left(\gamma^{\prime 2}+c d^{\prime 2}\right) /\left(\gamma^{\prime}+c d^{\prime}\right) .
$$


Similarly

$$
\langle\Delta E\rangle_{\mathrm{down}}=\int_{E^{\prime}=-\infty}^{E}\left(E^{\prime}-E\right) Z\left(E^{\prime}, E\right) d E^{\prime} / \int_{E^{\prime}=-\infty}^{E} Z\left(E^{\prime}, E\right) d E^{\prime}=-\left(\gamma^{2}+c d^{2}\right) /(\gamma+c d) .
$$

For the biexponential model, following a procedure similar to that in Sec. II B for the single exponential model, we obtain

$$
\frac{2 \pi i z(2 \pi i z-1 / k T)\left(2 \pi i z-r_{4}\right) \widetilde{g}_{-}(z)}{(1 / \gamma+2 \pi i z)(1 / d+2 \pi i z)}=-\frac{\left(1 / \gamma^{\prime}-2 \pi i z\right)\left(1 / d^{\prime}-2 \pi i z\right) \widetilde{g}_{+}(z)}{2 \pi i z-r_{3}},
$$

where

$$
r_{3}=1 / 2 k T \pm \sqrt{(1 / 2 k T)^{2}+\left[\left(\gamma+\gamma^{\prime}\right) / d d^{\prime}+\left(c d+c d^{\prime}\right) / \gamma \gamma^{\prime}\right] /\left(\gamma+\gamma^{\prime}+c d+c d^{\prime}\right)}
$$

and

$$
r_{4}=1 / 2 k T-\sqrt{(1 / 2 k T)^{2}+\left[\left(\gamma+\gamma^{\prime}\right) / d d^{\prime}+\left(c d+c d^{\prime}\right) / \gamma \gamma^{\prime}\right] /\left(\gamma+\gamma^{\prime}+c d+c d^{\prime}\right)}
$$

The solution for $\widetilde{g}_{-}(z)$ in Eq. (23), obtained in Appendix B, is

$$
\tilde{g}_{-}(z)=G_{b i} \frac{(1 / \gamma+2 \pi i z)(1 / d+2 \pi i z)}{2 \pi i z(2 \pi i z-1 / k T)\left(2 \pi i z-r_{4}\right)} .
$$

Inversion yields

$$
g(E)=g_{\mathrm{eq}}(0)\left[\mathrm{e}^{E / k T}+\frac{\gamma^{\prime} d^{\prime}}{\gamma d} \frac{1 / k t-r_{4}}{r_{4}}-\frac{\left(1 / \gamma+r_{4}\right)\left(1 / d+r_{4}\right) \gamma^{\prime} d^{\prime}}{r_{4} k t} \mathrm{e}^{-r_{4} E}\right] .
$$

This equation reduces to Eq. (19) when $d=\gamma$.

From these results, $k_{0}$ is given by

$$
k_{0}=Z_{0}\left[\gamma \gamma^{\prime}+c d d^{\prime}+\frac{\left(\gamma^{\prime 2}+c d^{2}\right) \gamma^{\prime} d^{\prime} r_{3}}{\gamma d r_{4}}-\left(\frac{1}{\gamma}+r_{4}\right)\left(\frac{1}{d}+r_{4}\right) \frac{\gamma^{\prime} d^{\prime}}{r_{4}}\left(\frac{\gamma^{\prime}}{1 / \gamma+r_{3}}+\frac{c d^{\prime}}{1 / d+r_{3}}\right)\right] g_{\mathrm{eq}}(0) \text {. }
$$

This equation reduces to Eq. (20) when $d=\gamma$.

\section{Singularity model}

The average up-energy transfer and down-energy transfer are given by

$$
\begin{aligned}
& \langle\Delta E\rangle_{\mathrm{up}}=\int_{E^{\prime}=E}^{\infty}\left(E^{\prime}-E\right) Z\left(E^{\prime}, E\right) d E^{\prime} / \int_{E^{\prime}=E}^{\infty} Z\left(E^{\prime}, E\right) d E^{\prime}=\gamma^{\prime}\left[1+C\left(\gamma^{\prime}\right)^{-\alpha}(1-\alpha) \Gamma(1-\alpha)\right] /\left[1+C\left(\gamma^{\prime}\right)^{-\alpha} \Gamma(1-\alpha)\right], \\
& \langle\Delta E\rangle_{\mathrm{down}}=\int_{E^{\prime}=-\infty}^{E}\left(E^{\prime}-E\right) Z\left(E^{\prime}, E\right) d E^{\prime} / \int_{E^{\prime}=-\infty}^{E} Z\left(E^{\prime}, E\right) d E^{\prime}=-\gamma\left[1+C(\gamma)^{-\alpha}(1-\alpha) \Gamma(1-\alpha)\right] /\left[1+C(\gamma)^{-\alpha} \Gamma(1-\alpha)\right] .
\end{aligned}
$$

To use a perturbation method for this case, although a suitable branch-point analysis might also be used, the $g(E)$ given by Eq. (20) and $Z\left(E^{\prime}, E\right)$ given by Eq. (12) are introduced into the right hand side of Eq. (7b). A new $g(E)$ is obtained, and this step is then iterated. After several iterations, we find that $g(E)$ for $E<-\gamma / 100$ ceases to be affected further. For $-\gamma / 100$ $<E \leq 0, g(E)$ becomes negligible because of continuity with $g(E)=0$ for $E>0$. Using this $g(E)$ to calculate $k_{0}$, we obtain a value close to the one obtained using $g(E)$ from Eq. (20), for $g(E)$ can be used here. The expression for the rate constant $k_{0}$ thus given by Eq. (5) is then

$$
\begin{aligned}
k_{0}= & Z_{0} \int_{E^{\prime}=0}^{\infty} \int_{E=-\infty}^{0} g_{\mathrm{eq}}(0)\left(\mathrm{e}^{-E / k T}-\frac{\gamma^{\prime}}{\gamma}\right) \mathrm{e}^{-\left(E^{\prime}-E\right) / \gamma^{\prime}}\left(1+C\left(E^{\prime}-E\right)^{-\alpha}\right) d E^{\prime} d E=Z_{0} g_{\mathrm{eq}}(0) \gamma^{\prime} \gamma\left(1-\frac{\gamma^{\prime 2}}{\gamma^{2}}\right) \\
& +C Z_{0} g_{\mathrm{eq}}(0) \int_{E^{\prime}=0}^{\infty} \int_{E=-\infty}^{0}\left(\mathrm{e}^{-E / k T}-\frac{\gamma^{\prime}}{\gamma}\right) \mathrm{e}^{-\left(E^{\prime}-E\right) / \gamma^{\prime}}\left(E^{\prime}-E\right)^{-\alpha} d E^{\prime} d E .
\end{aligned}
$$




\section{E. Near-singularity model}

For this model, the same functions are adopted for $Z\left(E^{\prime}, E\right)$ as in the singularity model. Because of the quantum limit, we set a lower bound $\varepsilon$ to the energy transfer in the integral when calculating the energy transfer and rate constant. Equation (27) then becomes

$$
\begin{aligned}
&\langle\Delta E\rangle_{\mathrm{up}}= \int_{E^{\prime}=E+\varepsilon}^{\infty}\left(E^{\prime}-E\right) Z\left(E^{\prime}, E\right) d E^{\prime} / \int_{E^{\prime}=E+\varepsilon}^{\infty} Z\left(E^{\prime}, E\right) d E^{\prime} \\
&= \frac{\int_{E^{\prime}=E+\varepsilon}^{\infty}\left(E^{\prime}-E\right)\left[1+C\left(E^{\prime}-E\right)^{-\alpha}\right] \exp \left(-\left(E^{\prime}-E\right) / \gamma^{\prime}\right) d E^{\prime}}{\int_{E^{\prime}=E+\varepsilon}^{\infty}\left[1+C\left(E^{\prime}-E\right)^{-\alpha}\right] \exp \left(-\left(E^{\prime}-E\right) / \gamma^{\prime}\right) d E^{\prime}}, \\
&\langle\Delta E\rangle_{\mathrm{down}}=\int_{E^{\prime}=-\infty}^{E-\varepsilon}\left(E^{\prime}-E\right) Z\left(E^{\prime}, E\right) d E^{\prime} / \int_{E^{\prime}=-\infty}^{E-\varepsilon} Z\left(E^{\prime}, E\right) d E^{\prime} \\
&=\frac{\int_{E^{\prime}=E+\varepsilon}^{\infty}\left(E^{\prime}-E\right)\left[1+C\left(E-E^{\prime}\right)^{-\alpha}\right] \exp \left(-\left(E-E^{\prime}\right) / \gamma\right) d E^{\prime}}{\int_{E^{\prime}=E+\varepsilon}^{\infty}\left[1+C\left(E-E^{\prime}\right)^{-\alpha}\right] \exp \left(-\left(E-E^{\prime}\right) / \gamma\right) d E^{\prime}},
\end{aligned}
$$

and we also have

$$
\begin{aligned}
k_{0}= & Z_{0}\left(\int_{E^{\prime}=\varepsilon}^{\infty} \int_{E=-\infty}^{0}+\int_{E^{\prime}=0}^{\varepsilon} \int_{E=-\infty}^{E^{\prime}-\varepsilon}\right) g_{\mathrm{eq}}(0)\left(\mathrm{e}^{-E / k T}-\frac{\gamma^{\prime}}{\gamma}\right) \mathrm{e}^{-\left(E^{\prime}-E\right) / \gamma^{\prime}}\left(1+C\left(E^{\prime}-E\right)^{-\alpha}\right) d E^{\prime} d E \\
= & Z_{\mathrm{eq}}(0)\left[\gamma^{\prime} \gamma\left(1-\frac{\gamma^{\prime 2}}{\gamma^{2}}\right) \mathrm{e}^{-\varepsilon / \gamma^{\prime}}-\gamma \gamma^{\prime}\left(k T+\frac{\varepsilon \gamma^{\prime}}{\gamma^{2}}\right) \mathrm{e}^{-\varepsilon / \gamma^{\prime}}+\gamma k T \mathrm{e}^{-\varepsilon / \gamma}\right]+C Z g_{\mathrm{eq}}(0)\left(\int_{E^{\prime}=\varepsilon}^{\infty} \int_{E=-\infty}^{0}+\int_{E^{\prime}=0}^{\varepsilon} \int_{E=-\infty}^{E^{\prime}-\varepsilon}\right) \\
& \times\left(\mathrm{e}^{-E / k T}-\frac{\gamma^{\prime}}{\gamma}\right) \mathrm{e}^{-\left(E^{\prime}-E\right) / \gamma^{\prime}}\left(E^{\prime}-E\right)^{-\alpha} d E^{\prime} d E .
\end{aligned}
$$

\section{APPLICATION TO $\mathrm{Ar}+\mathrm{O}_{3}$}

For the collisions of $\mathrm{O}_{3}$ with Ar, we obtain, from the trajectory data, ${ }^{40}$ the parameters for different temperatures and calculate $\langle\Delta E\rangle$ and $k_{0}$ for the single exponential model, the biexponential model, and the singularity model.

\section{A. Comparison of single exponential and biexponential models}

We determine $\gamma, c$, and $d$ from classical trajectory data, ${ }^{40}$ and $\gamma^{\prime}$ and $d^{\prime}$ are obtained from Eq. (11). The results for the $\langle\Delta E\rangle$ and $k_{0}$ for both models are given in Table I.

\section{B. Comparison of single exponential and singularity models}

We determine $C$ and $\alpha$ from the classical trajectory data. ${ }^{40}$ The average energy transfer and rate constants calculated from it are given in Table II.

\section{Comparison of single exponential and near-singularity models}

According to Ref. 40, collisional changes in $K$ provide a major route for the vibrational energy transfer. On that basis, a lower bound for the energy transfer is the quantum cutoff for the rotational energy $(A-B)\langle 2 K+1\rangle_{J}$, where $A$ and $B$ are the rotational constants and $K$ is the projection of the angular momentum along the principal axis of rotation, as noted in Sec. III A. An average over $J$ is used since we averaged over $J$ in the calculation of energy transfer. In Ref. 40, $A \sim 3.5 \mathrm{~cm}^{-1}$ and $B \sim 0.4 \mathrm{~cm}^{-1}$. From these values, the estimated lower bounds are given in Table III, namely from 15 to $30 \mathrm{~cm}^{-1}$, depending on the temperature. The resulting average energy transfer and rate constants are calculated and shown in Table III.

The cases $T=700 \mathrm{~K}$ and $T=1000 \mathrm{~K}$ are purely hypothetical since no experimental data are available for those conditions, only trajectory results ${ }^{40}$ are available for these temperatures. 
TABLE I. Example of correction of $k_{0},\langle\Delta E\rangle_{\text {up }}$, and $\langle\Delta E\rangle_{\text {down }}$. Single exponential model (s) and biexponential model (bi).

\begin{tabular}{llcc}
\hline \hline Parameters & $\frac{k_{0 b i} \text { a }}{k_{0 \mathrm{~s}}}$ & $\frac{\langle\Delta E\rangle_{\text {upbi b }}}{\langle\Delta E\rangle_{\text {ups }}}$ & $\frac{\left\langle\frac{\langle E\rangle_{\text {downbi b }}}{\langle\Delta E\rangle_{\text {downs }}}\right.}{}$ \\
\hline$\gamma=43 \mathrm{~cm}^{-1}{ }^{\mathrm{c}}, c=1.70^{\mathrm{c}}, d=3.70 \mathrm{~cm}^{-1}{ }^{\mathrm{c}}, T=298 \mathrm{~K}$ & 1.02 & 0.86 & 0.88 \\
$\gamma=119 \mathrm{~cm}^{-1}{ }^{\mathrm{c}}, c=3.27^{\mathrm{c}}, d=7.16 \mathrm{~cm}^{-1} \mathrm{c}, T=700 \mathrm{~K}$ & 1.01 & 0.82 & 0.84 \\
$\gamma=163 \mathrm{~cm}^{-1} \mathrm{c}^{\mathrm{c}}, c=3.49^{\mathrm{c}}, d=8.57 \mathrm{~cm}^{-1} \mathrm{c}, T=1000 \mathrm{~K}$ & 1.01 & 0.83 & 0.85 \\
\hline
\end{tabular}

${ }^{\mathrm{a}} k_{0 \mathrm{~s}}$ refers to the rate constant of the single exponential model and $k_{0 \mathrm{bi}}$ refers to the one of the biexponential model.

${ }^{\mathrm{b}} \mathrm{A}$ notation similar to that in footnote $\mathrm{b}$ is used for $\langle\Delta E\rangle_{\text {up }}$ and $\langle\Delta E\rangle_{\text {down }}$.

${ }^{c}$ Values of parameters were obtained using the trajectory results of $\mathrm{O}_{3} / \mathrm{Ar}$ collisions from Ref. 40.

\section{DISCUSSION}

For $\mathrm{Ar}+\mathrm{O}_{3}$, the $|\langle\Delta E\rangle|$ for the biexponential model is seen in Table I to be a little smaller than that from the single exponential model by about $10 \%-20 \%$. The $k_{0}$ for this biexponential model is about the same as that for the single exponential model (Table I). The $|\langle\Delta E\rangle|$ for the singularity model is seen in Table II to be smaller than that from the single exponential model by $20 \%-40 \%$. When we set a lower bound in $\Delta E$ for the singularity model, i.e., the nearsingularity model, and calculate the average up- and downenergy transfer, the results shown in Table III agree well with those from Ref. 40, as they should if this truncated singularity model is a good description of the trajectory data. The $k_{0}$ for the singularity and near-singularity models is larger than that for the single exponential model by a large factor, 25 , at room temperature, as seen in Tables II and III.

These results for $k_{0}$ can be interpreted in terms of the extra contribution from large $b$ collisions for the biexponential, singularity, and near-singularity models, compared with the single exponential model. The single exponential model is fitted to the low $b$ data. For the comparison of singularity and near-singularity models and the single exponential model, the difference for $\langle\Delta E\rangle_{\text {up/down }}$ is less than that for the $k_{0}$. This result can also be understood. $k_{0}$ is seen from Eq. (5) to have a larger contribution from small $|\Delta E|$ values to the integral than for the single exponential model. In the case of $|\langle\Delta E\rangle|$, the numerator in Eqs. (22), (27), and (29) is again enhanced by this enhanced $Z\left(E, E^{\prime}\right)$ but the denominator is enhanced even more, since the former is weighted by the small $\Delta E$.

In Refs. 11-13, the average down-energy refers to the total internal energy transfer instead of only vibrational energy transfer treated here. So that value should be much larger than that if only vibrational energy transfer were considered. If we compare the total internal down-energy transfer $\left\langle\Delta E_{\text {int }} \downarrow\right\rangle$ in Ivanov and Schinke's trajectory work ${ }^{40}$ with the values in Gao and Marcus' work ${ }^{11-13}$ used to fit experimental data, there is only a small difference in $\left\langle\Delta E_{\text {int }} \downarrow\right\rangle$.

The authors of Ref. 40 gave a different reason for choosing a cutoff of 3 or $10 \mathrm{~cm}^{-1}$ for $\left|\Delta E_{\text {int }}\right|$, namely that the energy transfer averages gradually decrease as the value for $\left|\Delta E_{\text {int }}\right|$ cutoff decreases and decrease particularly rapidly for the cutoff below the ones they chose. It was suggested that $b_{\max }$ may be found by weighting the average nenrgy transfer versus impact parameter by the differential cross section. ${ }^{63}$

Another result seen in Table I is that both $\gamma$ and $d$ $\left(\gamma^{\prime}\right.$ and $\left.d^{\prime}\right)$ for the trajectory data are proportional to $k T$, though are much smaller, and the ratios $d / \gamma$ and $d^{\prime} / \gamma^{\prime}$ remain almost the same. The increase in $d$ and $d^{\prime}$ with temperature means that small energy transfer behavior becomes less important at higher temperatures, as expected. The typical system is further removed from the singularity or $E^{\prime}-E=0$.

\section{CONCLUDING REMARKS}

Analytical solutions for the low pressure rate constant are given, using several different approximations to the trajectory data, the single exponential, a biexponential, a singularity, and a near-singularity models. The near-singularity model is the most realistic. The differences should be maximal in the low pressure regime. Expressions are obtained for the limiting low pressure rate constant $k_{0},\langle\Delta E\rangle_{\mathrm{up}}$, and $\langle\Delta E\rangle_{\text {down }}$. The values of $k_{0}$ from the biexponential are similar to those from the single exponential model. Those from the singularity and near-singularity models are an order of magnitude larger than those from the single exponential model. The origin of the difference is the large additional contribution of collisions with large cross sections in the singularity

TABLE II. Example of correction of $k_{0},\langle\Delta E\rangle_{\mathrm{up}}$, and $\langle\Delta E\rangle_{\mathrm{down}}$. Single exponential model and the singularity model.

\begin{tabular}{lccc}
\hline \hline Parameters & $\frac{k_{0 \text { singu }} \text { a }}{k_{0 \mathrm{~s}}}$ & $\frac{\langle\Delta E\rangle_{\text {upsingu }}}{\langle\Delta E\rangle_{\text {ups }}}$ & $\frac{\langle\Delta E\rangle_{\text {downsingu }}}{\langle\Delta E\rangle_{\text {downs }}}$ \\
\hline$\alpha=0.20^{\mathrm{c}}, \gamma=43 \mathrm{~cm}^{-1}{ }^{\mathrm{c}}, C=50^{\mathrm{c}}, T=298 \mathrm{~K}$ & 25.1 & 0.81 & 0.81 \\
$\alpha=0.35^{\mathrm{c}}, \gamma=119 \mathrm{~cm}^{-1}{ }^{\mathrm{c}}, C=50^{\mathrm{c}}, T=700 \mathrm{~K}$ & 10.8 & 0.67 & 0.67 \\
$\alpha=0.41^{\mathrm{c}}, \gamma=163 \mathrm{~cm}^{-1}{ }^{\mathrm{c}}, C=50^{\mathrm{c}}, T=1000 \mathrm{~K}$ & 7.6 & 0.63 & 0.63 \\
\hline
\end{tabular}

${ }^{\mathrm{a}} k_{0 \text { singu }}$ refers to the rate constant of the singularity model and $k_{0 \mathrm{~s}}$ refers to the single exponential model.

${ }^{\mathrm{b}} \mathrm{A}$ notation similar to that in footnote $\mathrm{b}$ is used for $\langle\Delta E\rangle_{\text {up }}$ and $\langle\Delta E\rangle_{\text {down }}$.

${ }^{c}$ Values of parameters were obtained using the trajectory results of $\mathrm{O}_{3} / \mathrm{Ar}$ collisions from Ref. 40. 
TABLE III. Example of cutoff effect for the near-singularity model.

\begin{tabular}{lccc}
\hline \hline Parameters & $\frac{k_{0 \text { n-singu }}}{k_{0 \mathrm{~s}}}$ & $\frac{\langle\Delta E\rangle_{\text {upn-singu }}}{\langle\Delta E\rangle_{\text {ups }}}$ & $\frac{\langle\Delta E\rangle_{\text {downn-singu }}}{\langle\Delta E\rangle_{\text {downs }}}$ \\
\hline$\alpha=0.20^{\mathrm{c}}, \gamma=43 \mathrm{~cm}^{-1}{ }^{\mathrm{c}}, C=50^{\mathrm{c}}, T=298 \mathrm{~K}$ & 24.9 & 1.06 & 1.06 \\
$\alpha=0.35^{\mathrm{c}}, \gamma=119 \mathrm{~cm}^{-1}{ }^{\mathrm{c}}, C=50^{\mathrm{c}}, T=700 \mathrm{~K}$ & 10.8 & 0.86 & 0.98 \\
$\alpha=0.41^{\mathrm{c}}, \gamma=163 \mathrm{~cm}^{-1}{ }^{\mathrm{c}}, C=50^{\mathrm{c}}, T=1000 \mathrm{~K}$ & 7.6 & 1.04 & 1.04 \\
\hline \hline
\end{tabular}

${ }^{\mathrm{a}} k_{0 \mathrm{~s}}$ refers to the rate constant of the single exponential model and $k_{0 \mathrm{n} \text {-singu }}$ refers to the rate constant of the near-singularity model with a lower bound. This lower bound is $15 \mathrm{~cm}^{-1}$ for $T=298 \mathrm{~K}, 25 \mathrm{~cm}^{-1}$ for $T$ $=700 \mathrm{~K}$, and $30 \mathrm{~cm}^{-1}$ for $T=1000 \mathrm{~K}$.

${ }^{\mathrm{b}} \mathrm{A}$ notation similar to that in footnote $\mathrm{b}$ is used for $\langle\Delta E\rangle_{\mathrm{up}}$ and $\langle\Delta E\rangle_{\text {down }}$.

${ }^{\mathrm{c}}$ Values of parameters were obtained using the trajectory results of $\mathrm{O}_{3} / \mathrm{Ar}$ collisions from Ref. 40.

and near-singularity models that is absent in the single exponential model. The values from single exponential model for the $\langle\Delta E\rangle$ are somewhat larger than those from the biexponential model by about $10 \%-20 \%$ and larger than those from the singularity model by $20 \%-40 \%$ but similar to those from the near-singularity model. The physical origin of these dif-

ferences lies in the smaller contribution from the smaller cross sections with large energy transfer in the biexponential and singularity models, compared with that in the single exponential model. While the numerator in Eqs. (22), (27), and (29) is enhanced by this enhanced $Z\left(E, E^{\prime}\right)$, the denominator is enhanced even more, since the former is weighted by the small $\Delta E$. For the near-singularity model, a big part of small energy transfer collision is removed. This cancels out the former effect.

\section{ACKNOWLEDGMENTS}

It is a pleasure to acknowledge the support of this research by the National Science Foundation and to thank Professor Jau Tang for a helpful discussion and Professor Reinhard Schinke and Dr. Mikhail V. Ivanov for providing their detailed classical trajectory data.

\section{APPENDIX A: SOLUTION OF EQ. (7b) AS A WIENER-HOPF EQUATION OF THE SECOND KIND FOR THE SINGLE EXPONENTIAL MODEL}

We first note that $\left|\widetilde{g}_{-}(z)\right|=\left|\int_{-\infty}^{\infty} \mathrm{e}^{2 \pi i z E} g_{-}(E) d E\right|$ and $\tilde{g}_{-}(z)$ is a function of $z$ alone. Also, $\left|\widetilde{g}_{-}(z)\right|$

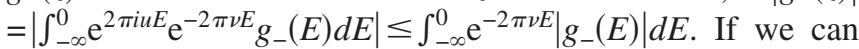
find a solution for $\widetilde{g}_{-}(z)$ such that $\left|g_{-}(E)\right|<M_{1} \mathrm{e}^{2 \pi u_{-} E}$, as $E \rightarrow-\infty$, where $\nu_{-}>0$ and hence tends to zero as $E \rightarrow-\infty$, then we have

$$
\left|\widetilde{g}_{-}(z)\right|<\int_{-\infty}^{0} \mathrm{e}^{-2 \pi \nu E} M_{1} \mathrm{e}^{2 \pi \nu_{-} E} d E=M_{1} \frac{1}{2 \pi\left(\nu_{-}-\nu\right)} .
$$

Thereby, in the part of the lower half plane where $\operatorname{Im} z=v<v_{-},\left|\widetilde{g}_{-}(z)\right|$ has no singularity. So $\widetilde{g}_{-}(z)$ is an analytic function in the half of the $z$-plane for which $\operatorname{Im} z<v_{-}$.

Next we consider $\tilde{g}_{+}(z)$,

$$
\left|\widetilde{g}_{+}(z)\right|=\left|\int_{-\infty}^{\infty} \mathrm{e}^{2 \pi i z E} g_{+}(E) d E\right|=\left|\int_{0}^{\infty} \mathrm{e}^{2 \pi i z E} g_{+}(E) d E\right|,
$$

since $g_{+}(E)=0$ for $E<0$. For $E>0$ it follows from Eq. (14c) that

$$
\begin{aligned}
\left|\widetilde{g}_{+}(z)\right|= & \mid \int_{0}^{\infty} \mathrm{e}^{2 \pi i z E} \\
& \times\left[\int_{-\infty}^{0} g_{-}\left(E^{\prime}\right) Z\left(E, E^{\prime}\right) d E^{\prime}\right] d E \mid / \int_{-\infty}^{\infty} Z\left(E^{\prime}, E\right) d E^{\prime} .
\end{aligned}
$$

We note that $\widetilde{g}_{+}(z)$ is a function of $z$ alone, and also from Eqs. (8a) and (A3),

$$
\begin{aligned}
\left|\widetilde{g}_{+}(z)\right|= & \mid \int_{0}^{\infty} \mathrm{e}^{2 \pi i u E} \mathrm{e}^{-2 \pi \nu E}\left[\int_{-\infty}^{0} g_{-}\left(E^{\prime}\right) \frac{1}{\gamma+\gamma^{\prime}} \mathrm{e}^{-E-E^{\prime} / \gamma^{\prime}} d E^{\prime}\right] \\
& \times d E\left|\leq \int_{0}^{\infty} \mathrm{e}^{-2 \pi \nu E}\right| \int_{-\infty}^{0} g_{-}\left(E^{\prime}\right) \frac{1}{\gamma+\gamma^{\prime}} \\
& \times \mathrm{e}^{-E-E^{\prime} / \gamma^{\prime}} d E^{\prime} \mid d E
\end{aligned}
$$

If we can find a solution $\left|g_{-}\left(E^{\prime}\right)\right|<M_{1} \mathrm{e}^{2 \pi u_{-} E^{\prime}}$, where $E^{\prime}<0$, then since $\nu_{-}>0$ we have $\nu_{-}>-\frac{1}{2 \pi \gamma^{\prime}}$ and then

$$
\begin{aligned}
\left|\widetilde{g}_{+}(z)\right| & \leq \int_{0}^{\infty} \mathrm{e}^{-2 \pi \nu E}\left|\int_{-\infty}^{0} g_{-}\left(E^{\prime}\right) \frac{1}{\gamma+\gamma^{\prime}} \mathrm{e}^{-E-E^{\prime} / \gamma^{\prime}} d E^{\prime}\right| d E \\
& =\frac{1}{\gamma+\gamma^{\prime}} \frac{1}{2 \pi \nu+1 / \gamma^{\prime}}\left|\int_{-\infty}^{0} g_{-}\left(E^{\prime}\right) \mathrm{e}^{E^{\prime} / \gamma^{\prime}} d E^{\prime}\right| \\
& <\frac{M_{1}}{\gamma+\gamma^{\prime}} \frac{1}{2 \pi \nu+1 / \gamma^{\prime}} \frac{1}{2 \pi \nu_{-}+1 / \gamma^{\prime}} .
\end{aligned}
$$

Thereby, $\left|\widetilde{g}_{+}(z)\right| \rightarrow 0$ as $\operatorname{Im} z=\nu \rightarrow \infty$, and we see that $\tilde{g}_{+}(z)$ has no singularity in the upper half plane for which $\operatorname{Im} z>-\left(1 / 2 \pi \gamma^{\prime}\right)$, and so is analytic in that upper half plane. The right-hand side of Eq. (17) is therefore analytic in the upper half plane, $\operatorname{Im} z>-\left(1 / 2 \pi \gamma^{\prime}\right)$. We had seen earlier in Appendix A that $\widetilde{g}_{-}(z)$ is analytic in the lower half plane $\operatorname{Im} z<v_{-}$. From the derivation on the left-hand side of Eq. (17), we need for analyticity of this side of the equation, $\operatorname{Im} z<-1 / 2 \pi k T$. Thereby, we require that the left-hand side of Eq. (17) is analytic in the lower half plane where $\operatorname{Im} z<-(1 / 2 \pi k T)$. Since $v_{-}>0>-\left(1 / 2 \pi \gamma^{\prime}\right)$ we now see 
that both sides of Eq. (17) are analytic in the strip $-\left(1 / 2 \pi \gamma^{\prime}\right)<\operatorname{Im} z<-(1 / 2 \pi k T)$. Since the analytic continuation is unique, there exists an entire function $F$ in the complex plane which coincides with the right-hand side of Eq. (17) in upper half plane $\operatorname{Im} z>-\left(1 / 2 \pi \gamma^{\prime}\right)$ and coincides with the left-hand side of Eq. (17) in the lower half plane $\operatorname{Im} z<\operatorname{Min}\left(-(1 / 2 \pi k T), v_{-}\right)$. Since $\tilde{g}_{+}(z)$ goes to zero, no slower than exponentially as $|z| \rightarrow \infty$, then the entire function $F$ is bounded at infinity. One concludes that $F$ is constant, which we denote by $G_{s}$, and so obtain

$$
\begin{aligned}
& \tilde{g}_{-}(z)=G_{s} \frac{(1 / \gamma+2 \pi i z)}{2 \pi i z(2 \pi i z-1 / k T)}, \\
& \tilde{g}_{+}(z)=G_{s} \frac{\gamma+\gamma^{\prime}}{1 / \gamma^{\prime}-2 \pi i z} .
\end{aligned}
$$

\section{APPENDIX B: SOLUTION OF EQ. (7b) \\ AS A WIENER-HOPF EQUATION OF THE SECOND KIND FOR THE BIEXPONENTIAL MODEL}

Following similar procedure in Appendix A, we can now solve Eq. (23). As in Appendix A, $\left|\widetilde{g}_{-}(z)\right|$ $=\left|\int_{-\infty}^{\infty} \mathrm{e}^{2 \pi i z E} g_{-}(E) d E\right|$, and $\tilde{g}_{-}(z)$ is a function of $z$ alone. Also,

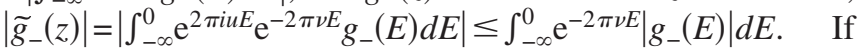
we can find a solution for $\tilde{g}_{-}(z)$ such that $\left|g_{-}(E)\right|$ $<M_{1} \mathrm{e}^{2 \pi v_{-} E}$, as $E \rightarrow-\infty$, where $\nu_{-}>0$ and hence tends to zero as $E \rightarrow-\infty$. Then we have

$$
\left|\widetilde{g}_{-}(z)\right|<\int_{-\infty}^{0} \mathrm{e}^{-2 \pi \nu E} M_{1} \mathrm{e}^{2 \pi \nu_{-} E} d E=M_{1} \frac{1}{2 \pi\left(\nu_{-}-\nu\right)} .
$$

Thereby, $\left|\widetilde{g}_{-}(z)\right|$ has no singularity in the part of the lower half plane where $\operatorname{Im} z=v<v_{-}$. So $\widetilde{g}_{-}(z)$ is analytic in the half of the $z$-plane for which $\operatorname{Im} z<v_{-}$.

Next we consider $\widetilde{g}_{+}(z)$,

$$
\left|\widetilde{g}_{+}(z)\right|=\left|\int_{-\infty}^{\infty} \mathrm{e}^{2 \pi i z E} g_{+}(E) d E\right|=\left|\int_{0}^{\infty} \mathrm{e}^{2 \pi i z E} g_{+}(E) d E\right| .
$$

Since $g_{+}(E)=0$ for $E<0$, for $E>0$ it follows from Eq. (14c) that

$$
\left|\widetilde{g}_{+}(z)\right|=\left|\int_{0}^{\infty} \mathrm{e}^{2 \pi i z E}\left[\int_{-\infty}^{0} g_{-}\left(E^{\prime}\right) Z\left(E, E^{\prime}\right) d E^{\prime}\right] d E / \int_{-\infty}^{\infty} Z\left(E^{\prime}, E\right) d E^{\prime}\right| .
$$

We note that $\widetilde{g}_{+}(z)$ is a function of $z$ alone, and also from Eq. (9a)

$$
\begin{aligned}
\left|\widetilde{g}_{+}(z)\right| & =\left|\int_{0}^{\infty} \mathrm{e}^{2 \pi i u E} \mathrm{e}^{-2 \pi \nu E}\left[\int_{-\infty}^{0} g_{-}\left(E^{\prime}\right) \frac{1}{\gamma+\gamma^{\prime}+c d+c d^{\prime}}\left(\mathrm{e}^{-E-E^{\prime} / \gamma^{\prime}}+c \mathrm{e}^{-E-E^{\prime} / d^{\prime}}\right) d E^{\prime}\right] d E\right| \\
& \leq \int_{0}^{\infty} \mathrm{e}^{-2 \pi \nu E}\left|\int_{-\infty}^{0} g_{-}\left(E^{\prime}\right) \frac{1}{\gamma+\gamma^{\prime}+c d+c d^{\prime}}\left(\mathrm{e}^{-E-E^{\prime} / \gamma^{\prime}}+c \mathrm{e}^{-E-E^{\prime} / d^{\prime}}\right) d E^{\prime}\right| d E .
\end{aligned}
$$

If, as stated above, we can find a solution $\left|g_{-}\left(E^{\prime}\right)\right|<M_{1} \mathrm{e}^{2 \pi v_{-} E^{\prime}}$, where $E^{\prime}<0$, then

$$
\begin{aligned}
\left|\widetilde{g}_{+}(z)\right| & \leq \int_{0}^{\infty} \mathrm{e}^{-2 \pi \nu E}\left|\int_{-\infty}^{0} g_{-}\left(E^{\prime}\right) \frac{1}{\gamma+\gamma^{\prime}+c d+c d^{\prime}}\left(\mathrm{e}^{-E-E^{\prime} / \gamma^{\prime}}+c \mathrm{e}^{-E-E^{\prime} / d^{\prime}}\right) d E^{\prime}\right| d E \\
& =\frac{1}{\gamma+\gamma^{\prime}+c d+c d^{\prime}}\left|\frac{1}{2 \pi \nu+1 / \gamma^{\prime}} \int_{-\infty}^{0} g_{-}\left(E^{\prime}\right) \mathrm{e}^{E^{\prime} / \gamma^{\prime}} d E^{\prime}+\frac{1}{2 \pi \nu+1 / d^{\prime}} \int_{-\infty}^{0} g_{-}\left(E^{\prime}\right) \mathrm{e}^{E^{\prime} / d^{\prime}} d E^{\prime}\right| \\
& <\frac{M_{1}}{\gamma+\gamma^{\prime}+c d+c d^{\prime}}\left(\frac{1}{2 \pi \nu+1 / \gamma^{\prime}} \frac{1}{2 \pi \nu_{-}+1 / \gamma^{\prime}}+\frac{1}{2 \pi \nu+1 / d^{\prime}} \frac{1}{2 \pi \nu_{-}+1 / d^{\prime}}\right) .
\end{aligned}
$$

Thereby, $\left|\tilde{g}_{+}(z)\right| \rightarrow 0$ as $\operatorname{Im} z=\nu \rightarrow \infty$, and $\tilde{g}_{+}(z)$ has no singularity in the upper half plane for which $\operatorname{Im} z>-\left(1 / 2 \pi \gamma^{\prime}\right)$ $>-\left(1 / 2 \pi d^{\prime}\right)$ and since $\nu_{-}>0>-\left(1 / 2 \pi \gamma^{\prime}\right)>-\left(1 / 2 \pi d^{\prime}\right)$, it is analytic in that upper half plane. The right-hand side of Eq. (23) is therefore analytic in the upper half plane for which this condition is fulfilled and where

$$
\operatorname{Im} z>\max \left(-\left(1 / 2 \pi \gamma^{\prime}\right),\left(-r_{3} / 2 \pi\right)\right)
$$

where

$$
r_{3}=1 / 2 k T+\sqrt{(1 / 2 k T)^{2}+\left[\left(\gamma+\gamma^{\prime}\right) / d d^{\prime}+\left(c d+c d^{\prime}\right) / \gamma \gamma^{\prime}\right] /\left(\gamma+\gamma^{\prime}+c d+c d^{\prime}\right)} .
$$


We had seen earlier in Appendix B that $\widetilde{g}_{-}(z)$ is analytic in the lower half plane $\operatorname{Im} z<v_{-}$. From the derivation on the left-hand side of Eq. (23), we need for analyticity of this side of the equation $\operatorname{Im} z<-1 / 2 \pi k T$. Thereby, we require that the left-hand side of Eq. (23) is analytic in the lower half plane where $\operatorname{Im} z<-(1 / 2 \pi k T)$. Since $v_{-}>0>-\left(1 / 2 \pi \gamma^{\prime}\right)$, we now see that both sides of Eq. (23) are analytic in the strip $\max \left(-\left(1 / 2 \pi \gamma^{\prime}\right),\left(-r_{3} / 2 \pi\right)\right)<\operatorname{Im} z<-(1 / 2 \pi k T)$. Since the analytic continuation is unique, there exists an entire function $F$ in the complex plane which coincides with the right-hand side of Eq. (23) in upper half plane $\operatorname{Im} z>\max \left(-\left(1 / 2 \pi \gamma^{\prime}\right),\left(-r_{3} / 2 \pi\right)\right)$ and coincides with the left-hand side of Eq. (23) in the lower half plane $\operatorname{Im} z<-(1 / 2 \pi k T)$. Since $\tilde{g}_{+}(z)$ goes to zero no slower than exponentially as $|z| \rightarrow \infty$, then the entire function $F$ should be bounded at infinity. One concludes that $F$ is constant, which we denote by $G_{b i}$, and so obtain

$$
\tilde{g}_{-}(z)=G_{b i} \frac{(1 / \gamma+2 \pi i z)(1 / d+2 \pi i z)}{2 \pi i z(2 \pi i z-1 / k T)\left(2 \pi i z-r_{4}\right)} .
$$

${ }^{1}$ D. C. Tardy and B. S. Rabinovitch, J. Chem. Phys. 45, 3720 (1966).

${ }^{2}$ R. C. Bhattacharjee and W. Forst, Sym. (Int.) Combust., [Proc.] 15, 681 (1975).

${ }^{3}$ W. G. Valance and E. W. Schlag, J. Chem. Phys. 45, 4280 (1966).

${ }^{4}$ J. E. Dove, W. S. Nip, and H. Teitelbaum, Sym. (Int.) Combust., [Proc.] 15, 689 (1975).

${ }^{5}$ J. Troe, J. Chem. Phys. 66, 4745 (1977).

${ }^{6}$ R. E. Weston, Jr., Chem. Rev. 99, 2115 (1999).

${ }^{7}$ M. H. Thiemens and H. E. Heidenreich, Science 219, 1073 (1983).

${ }^{8}$ J. Yang and S. Epstein, Geochim. Cosmochim. Acta 51, 2011 (1987).

${ }^{9}$ K. Mauersberger, Geophys. Res. Lett. 14, 80, DOI: 10.1029/ GL014i001p00080 (1987)

${ }^{10}$ D. Krankowsky, P. Lammerzahl, and K. Mauersberger, Geophys. Res. Lett. 27, 2593, DOI: 10.1029/2000GL011812 (2000).

${ }^{11}$ Y. Q. Gao and R. A. Marcus, Science 293, 259 (2001).

${ }^{12}$ Y. Q. Gao and R. A. Marcus, J. Chem. Phys. 116, 137 (2002).

${ }^{13}$ Y. Q. Gao and R. A. Marcus, J. Chem. Phys. 117, 1536 (2002)

${ }^{14}$ B. C. Hathorn and R. A. Marcus, J. Chem. Phys. 111, 4087 (1999).

${ }^{15}$ J. Morton, J. Barnes, B. Schueler, and K. Mauerberger, J. Geophys. Res. 95, 901, DOI: 10.1029/JD095iD01p00901 (1990).

${ }^{16}$ M. H. Thiemens and T. Jackson, Geophys. Res. Lett. 17, 717, DOI: 10.1029/GL017i006p00717 (1990).

${ }^{17}$ K. Mauersberger, B. Erbacher, D. Krankowsky, J. Gunther, and R. Nickel, Science 283, 370 (1999).

${ }^{18}$ D. Charlo and D. C. Clary, J. Chem. Phys. 120, 2700 (2004).

${ }^{19}$ R. Schinke, S. Y. Grebenshchikov, M. V. Ivanov, and P. Fleurat-Lessard, Annu. Rev. Phys. Chem. 57, 625 (2006).

${ }^{20}$ D. Babikov, B. K. Kendrick, R. B. Walker, P. R. Fleurat-Lessard, and R. Schinke, J. Chem. Phys. 119, 2577 (2003).

${ }^{21}$ B. C. Hathorn and R. A. Marcus, J. Chem. Phys. 113, 9497 (2000).

${ }^{22}$ C. Janssen, J. Guenther, J. K. Mauersberger, and D. Krankowsky, Phys. Chem. Chem. Phys. 3, 4718 (2001).

${ }^{23}$ G. H. Kohlmaier and B. S. Rabinovitch, J. Chem. Phys. 38, 1692 (1963).

${ }^{24}$ J. W. Simons, B. S. Rabinovitch, and D. W. Setser, J. Chem. Phys. 41, 800 (1964).
${ }^{25}$ U. Hold, T. Lenzer, K. Luther, K. Reihs, and A. Symonds, Ber. Bunsenges. Phys. Chem 101, 552 (1997).

${ }^{26}$ N. J. Brown and J. A. Miller, J. Chem. Phys. 80, 5568 (1984).

${ }^{27}$ X. Hu and W. L. Hase, J. Phys. Chem. 92, 4040 (1988).

${ }^{28} \mathrm{H}$. Hippler and J. Troe, in Advances in Gas Phase Photochemistry and Kinetics-Biomolecular Collisions, edited by M. N. R. Ashfold and J. E. Baggott (The Chemical Society, London, 1989).

${ }^{29}$ D. L. Clarke, I. Oref, and R. G. Gilbert, J. Chem. Phys. 96, 5983 (1992).

${ }^{30}$ K. F. Lim and R. G. Gilbert, J. Phys. Chem. 94, 72 (1990).

${ }^{31}$ A. Gelb, J. Phys. Chem. 89, 4189 (1985).

${ }^{32}$ H. Hippler, H. W. Schranz, and J. Troe, J. Phys. Chem. 90, 6158 (1986).

${ }^{33}$ I. Koifman, E. I. Dashevskaya, E. E. Nikitin, and J. Troe, J. Phys. Chem. 99, 15348 (1995).

${ }^{34}$ M. V. Ivanov, S. Y. Grebenshchikov, and R. Schinke, J. Chem. Phys. 120, 10015 (2004).

${ }^{35}$ A. J. Stace and J. N. Murrell, J. Chem. Phys. 68, 3028 (1978).

${ }^{36}$ A. J. Stace and J. N. Murrell, J. Chem. Soc., Faraday Trans. 74, 2182 (1965).

${ }^{37}$ I. Oppenheim, K. E. Shuler, and G. Weiss, Adv. Mol. Relax. Processes 1, 13 (1967).

${ }^{38}$ A. P. Penner and W. J. Forst, J. Chem. Phys. 67, 5296 (1977).

${ }^{39}$ R. G. Gilbert and S. C. Smith, Theory of Unimolecular and Recombination Reactions (Blackwell Scientific, Oxford, 1990).

${ }^{40}$ M. V. Ivanov and R. Schinke, J. Chem. Phys. 122, 234318 (2005).

${ }^{41}$ N. J. Brown and J. A. Miller, J. Chem. Phys. 80, 5568 (1984).

${ }^{42}$ L. M. Yoder and J. R. Barker, J. Phys. Chem. A 104, 10184 (2000).

${ }^{43}$ J. R. Barker, L. M. Yoder, and K. D. King, J. Phys. Chem. A 105, 796 (2001).

${ }^{44}$ S. C. Smith and R. G. Gilbert, Int. J. Chem. Kinet. 20, 307 (1988).

${ }^{45}$ J. A. Miller, S. J. Klippenstein, and C. Raffy, J. Phys. Chem. A 106, 4904 (2002)

${ }^{46}$ J. Keck and G. J. Carrier, J. Chem. Phys. 43, 2284 (1965).

${ }^{47}$ S. H. Robertson, M. J. Pilling, D. L. Baulch, and N. J. B. Green, J. Phys. Chem. 99, 13452 (1995) (and references cited therein).

${ }^{48}$ S. H. Robertson, M. J. Pilling, K. E. Gates, and S. C. Smith, J. Comput. Chem. 18, 1004 (1997).

${ }^{49}$ W. D. Lawrance, A. E. W. Knight, R. G. Gilbert, and K. D. King, Chem. Phys. Lett. 76, 113 (1980) (and references cited therein).

${ }^{50}$ S. Nordholm, L. E. B. Börjesson, L. Ming, and H. Svedung, Ber. Bunsenges. Phys. Chem 101, 574 (1997) (and references cited therein).

${ }^{51}$ M. J. Pilling and S. H. Robertson, Annu. Rev. Phys. Chem. 54, 245 (2003).

${ }^{52}$ B. J. Gaynor, R. G. Gilbert, and K. D. King, Chem. Phys. Lett. 55, 40 (1978) (and references cited therein).

${ }^{53}$ A. P. Penner and W. Forst, J. Chem. Phys. 67, 5296 (1977).

${ }^{54}$ T. Kato, J. Chem. Phys. 108, 6611 (1998).

${ }^{55}$ T. J. Frankcombe and S. C. Smith, J. Comput. Chem. 21, 592 (2000).

${ }^{56}$ K. A. Holbrook, M. J. Pilling, and S. H. Robertson, Unimolecular Reactions, 2nd ed. (Wiley, New York, 1996).

${ }^{57}$ S. Nordholm and H. W. Schranz, in Advances in Chemical Kinetics and Dynamics, Vol. 2A, edited by J. R. Barker (JAI, Connecticut, 1995).

${ }^{58}$ S. H. Kang and K.-H. Jung, Chem. Phys. Lett. 131, 496 (1986).

${ }^{59}$ H. O. Pritchard, J. Phys. Chem. A 108, 5249 (2004).

${ }^{60} \mathrm{~W}$. Forst, Theory of Unimolecular Reactions (Academic, New York, London, 1973).

${ }^{61}$ A. D. Polyanin and A. V. Manzhirov, Handbook of Integral Equations (CRC, Boca Raton, FL, 1998).

${ }^{62}$ A. R. Whyte, K. F. Lim, and R. G. Gilbert, Chem. Phys. Lett. 152, 377 (1988).

${ }^{63}$ O. Meroueh and W. L. Hase, Int. J. Mass Spectrom. 201, 233 (2000). 